\title{
Epidemiologia do araneísmo no município de Chapecó, Santa Catarina, Brasil
}

\section{Clinical and epidemiological features of araneism in the city of Chapecó, State of Santa Catarina, Brazil}

\author{
Fernanda Lise ${ }^{1}$; Flávio Roberto Mello Garcia ${ }^{2}$
}

\begin{abstract}
Resumo
Este estudo, de caráter descritivo e exploratório, foi realizado com o objetivo de verificar a incidência e caracterizar dos acidentes com aranhas de 1995 a 2002 no município de Chapecó, Santa Catarina, bem como caracterizar esses acidentes. Para tanto, verificaram-se os registros das notificações realizadas pela Vigilância Epidemiológica de Chapecó entre os anos e calculou-se a freqüência das mesmas. Em Chapecó, ocorreram 131 acidentes com aranhas durante o período estudado, e a incidência de araneísmo foi de $0,8 \%$ em $1995 ; 0,0 \%$ em 1996; 2,2\% em 1997; 8,7\% em 1998; 11,9\% em 1999; 18,4\% em 2000; 17,5\% em 2001 e de 29,8\% em 2002. Dentre eles, 62,6\% foram ocasionados por aranhas do gênero Loxosceles. A maioria ocorreu com mulheres e adultos entre 21 e 60 anos (54,9\%). Cerca de $80 \%$ dos acidentes ocorreram na área urbana, visto que quase $80 \%$ das vítimas residiam nesta área. As partes do corpo mais picadas pelas aranhas foram coxas/pernas, pé/artelhos e mão/dedos. As principais alterações clínicas apresentadas pelos pacientes foram dor, edema e eritema. A estação de maior ocorrência de acidentes foi a primavera, e as vítimas em mais de $40 \%$ encontravam-se em atividades no interior das residências. Conclui-se que o número de acidentes vem aumentando significativamente nos últimos anos, e isso demonstra a necessidade de políticas públicas de saúde para a prevenção destes.

Palavras-chave: Loxoscelismo. Aranhas. Epidemiologia.
\end{abstract}

\begin{abstract}
This descriptive and exploratory study was carried out in order to verify the incidence of and characterize accidents with spiders, from 1995 to 2002, in the city of Chapecó, State of Santa Catarina. Notification records kept by the Center for Epidemiologic Surveillance of Chapecó concerning that period of time were analyzed, and the number of occurrences was calculated. In Chapecó, 131 accidents with spiders occurred during that period of time, and the incidence of araneism was of $0.8 \%$ in 1995; $0.0 \%$ in 1996; $2,2 \%$ in $1997 ; 8.66 \%$ in $1998 ; 11.9 \%$ in $1999 ; 18,4 \%$ in $2000 ; 17,5 \%$ in 2001 and $29,8 \%$ in 2002. Sixty-two point six per cent $(62,6 \%)$ of these accidents were caused by spider of the genera Loxosceles. Most of them occurred with women and adults from 21 to 60 years old (54,9\%). Approximately $80 \%$ of the accidents occurred in the urban area, since $80 \%$ of the victims lived in the city. The parts of the body that were most bitten by spiders were thigh/legs, foot/toes and hand/fingers. The main clinical alterations presented by the patients were pain, edema and erythema. The season when most accidents occurred was the Spring, and more than $40 \%$ of the victims were doing housework . Results from this study showed that the number of accidents has increased significantly in recent years, and that the implementation of public health policies in this area to prevent such accidents to happen is necessary.

Key words: Loxoscelism. Spiders. Epidemiology.
\end{abstract}

${ }^{1}$ Enfermeira, Especialista em Enfermagem Pediátrica. Universidade Federal do Rio Grande do Sul. Escola de Enfermagem. Rua: São Manoel 963, Porto Alegre, RS - CEP. 90620-110.

2 Biólogo, Prof. Dr. do UNILASALLE, e do Programa de Mestrado em Ciências Ambientais da UNOCHAPECÓ. Coordenação de Pós-graduação Strictu sensu e Pesquisa; Av. Victor Barreto, 2288, CEP 92010-000, Canoas, RS. E-mail: frmg @ unilasalle.edu.br. Fone: (51) 3476-8624. 


\section{Introdução}

No Brasil, as espécies de aranhas que podem ocasionar acidentes pertencem aos gêneros Latrodectus (viúva negra), Loxosceles (aranha marrom) e Phoneutria (armadeira) perfazendo um total de cerca de 20 espécies (LUCAS; SILVA JUNIOR, 1992). Esses gêneros são responsáveis por $81 \%$ dos acidentes por aranhas (BARBARO; JARED; MOTA, 1995), sendo notificados cerca de 5.000 acidentes anualmente no país (BOCHNER; STRUCHINER, 2002). Os acidentes com Latrodectus ocorrem na maioria das vezes com trabalhadores nas colheitas de trigo e linho. Os acidentes com Phoneutria (foneutrismo) ocorrem principalmente durante o período diurno (BUCHARETCHI, 1992) enquanto os ocasionados por Loxosceles (loxoscelismo) ocorrem à noite, e as aranhas desse grupo alojam-se em lugares quentes e secos, sempre ao abrigo da luz (CAMPOS; GARCIA, 1997; CUPO; AZEVEDO-MARQUES; HERING, 2003). Loxosceles intermedia (Mello Leitão, 1934) foi registrada pela primeira vez no município de Chapecó em residências nas áreas rural e urbana do município por Campos e Garcia (1997).

O número de notificações de acidentes provocados por aranhas têm aumentado nos últimos anos, principalmente a partir de 1998 (BOCHNER; STRUCHINER, 2002), e a maioria deles se concentra nos Estados do Sul, particularmente no Paraná e Santa Catarina. O Estado do Paraná é o estado brasileiro que notifica o maior número de acidentes por aranhas do gênero Loxoceles no país (MARQUES-DA-SILVA; FISCHER, 2005), visto que, se tem aumentado a divulgação e o conhecimento sobre este tipo de acidente e, ainda uma iniciativa do Ministério da Saúde de tornar obrigatória a notificação dos casos de araneísmo (BRAsIL, 1999).

Estes são classificados em leves, moderados e graves. No primeiro caso, os principais sintomas são dor, irradiação da dor, parestesia, sudorese, hiperemia, edema e o sinal da picada; como tratamento geral ,indica-se a observação clínica, anestésico local e/ou analgésico. Os sintomas dos acidentes classificados como moderados são a taquicardia, hipertensão arterial, sudorese profusa, agitação psicomotora, visão turva, vômitos ocasionais, dor abdominal, priapismo e sialorréia discreta; o tratamento geral é internação hospitalar, anestésico local e/ou analgésico, além de soro antiaracnídico 2-4 ampolas IV (Intra-Venoso). No caso de acidentes graves, os principais sintomas são vômitos profusos e freqüentes, bradicardia, hipotensão arterial, insuficiência cardíaca, arritmias cardíacas, choque, dispnéia, coma, convulsões, edema pulmonar agudo e parada cardiorespiratória; o tratamento indicado é internação em Unidade de Cuidados Intensivos e soro antiaracnídico 5-10 ampolas IV. (LUCAS; SILVA JUNIOR, 1992; BRASIL, 1998).

Este estudo foi realizado com o objetivo de verificar a incidência e as características dos acidentes com aranhas de 1995 a 2002, no município de Chapecó, Santa Catarina.

\section{Material e método}

O estudo foi de caráter descritivo e exploratório. Para tanto, verificaram-se todas as notificações de acidentes com aranhas na Secretaria Municipal de Saúde, Departamento de vigilância epidemiológica do Município de Chapecó nos anos 1995 a 2002. A coleta dos dados fora realizada pela primeira autora, e como instrumento para a coleta de dados utilizouse um questionário estruturado com os seguintes itens: data do acidente; identificação da vítima (sexo e idade): zona de residência e de ocorrência do acidente: período do ano, local do corpo que esteve em contato, data do atendimento, tempo decorrido do contato ao atendimento, alterações clínicas no local de contato, complicações clínicas em geral, classificação do acidente, utilização de soroterapia, espécie de aranha: e evolução do contato.

Para a população de 1995, utilizou-se o dado do senso do IBGE de 1991, acrescentando crescimento de $3,35 \%$ ao ano, já para os anos seguintes foi 
utilizado o senso de 1996 e 2000 com crescimento anual de 2,84\%. Com esse procedimento, buscou-se corrigir o número de acidentes com o da população residente no Município (IBGE, 2003).

Efetuou-se cálculo de frequência $(\mathrm{F} \%)$ para cada item do instrumento de pesquisa, conforme a equação a seguir:

$$
\mathbf{F} \%=\frac{\text { Tipo resposta } \times 100}{\text { Total de resposta do item }}
$$

\section{Resultados e discussão}

A incidência dos casos de araneísmo em Chapecó foi de $0,8 \%$ em 1995; 0,0\% em 1996; 2,2\% em 1997; $8,7 \%$ em $1998 ; 11,9 \%$ em $1999 ; 18,4 \%$ em 2000; 17,5\% em 2001 e de 29\% em 2002 (Figura 1), demonstrando o crescimento do número de notificações de acidentes no Município, o que possibilita um melhor dimensionamento desse tipo de agravo nas diversas regiões do país (BRASIL, 1999).
No município de Chapecó ocorreram 131 casos de acidentes com aranhas, durante o período estudado, dos quais $62,6 \%$ ocasionados por aranhas do gênero Loxosceles, possivelmente grande parte por Loxosceles intermedia, dada à abundância dessa espécie no Município (CAMPOS; GARCIA, 1997). Ainda na Região Oeste de Santa Catarina no Município de São Miguel do Oeste, $60 \%$ dos acidentes com aranhas no ano de 2003, foram ocasionados pelo gênero Loxoceles (BARP; GARCIA, 2005), dado que corresponde à forma mais grave de araneísmo na América do Sul (CARDOSO, 1992).

O Phoneutrismo foi representado pôr 8,4\%, possivelmente ocasionado pela espécie Phoneutria nigriventer, que é a espécie mais comum na região. Salienta-se o número elevado de identificações ignoradas ocorridas, possivelmente pela não coleta e envio do espécime de aranha pela vítima, (haja vista que em apenas metade dos casos as aranhas são levadas para a identificação (BUCHARETCHI, 1992), ou pela danificação do espécime levado.

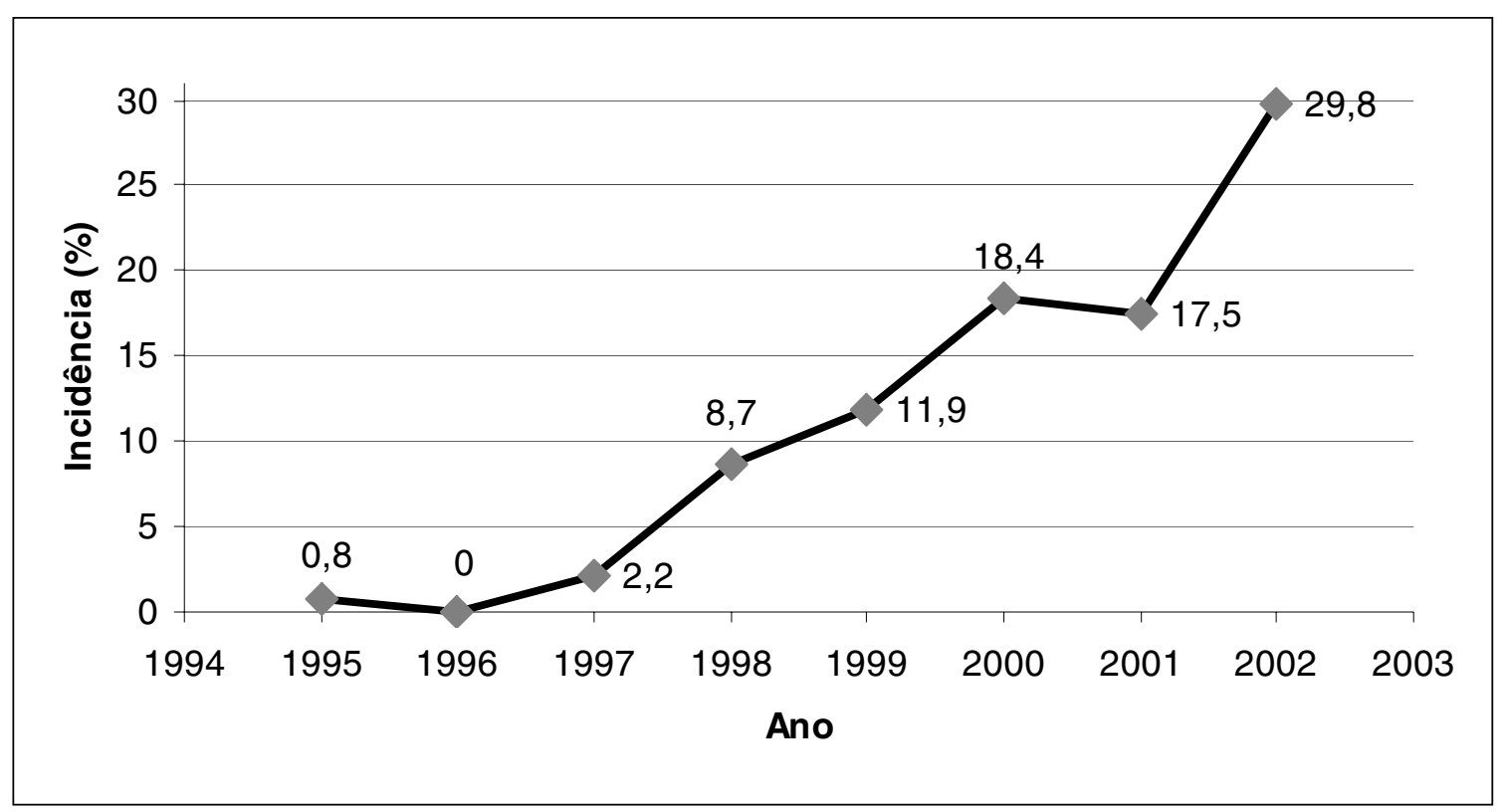

Figura 1. Incidência do número de casos de araneísmo de 1995 a 2002 no município de Chapecó, SC. 
A maioria dos acidentes ocorreu com mulheres (54\%) e pessoas com idades entre 21 e 60 anos $(55 \%)$. No Brasil, a maioria dos acidentes ocorre com adultos, predominantemente com em mulheres, possivelmente pela maior ocorrência de Loxosceles no interior das residências, principalmente dentro de roupas, sapatos, toalhas e outros utensílios (BRASIL, 1999; CAMPOS; GARCIA, 1997), fato de possibilitar o maior contato de donas de casa com estes animais. Mais de $80 \%$ dos acidentes com aranhas ocorreram na área urbana, visto que quase $80 \%$ das vítimas residiam nesta área. A estação do ano de maior ocorrência de acidentes foi a primavera (40\%), corroborando os resultados obtidos por Schenone (2003) em estudo com 250 registros de acidentes em Santiago do Chile, e diferindo dos resultados de Bucharetchi (1992), Cardoso (1992) e Málaque et al. (2002), que, no Estado de São Paulo, verificaram maior número de acidentes no verão. A diferença no período de atividade observados nos Estados de São Paulo e Santa Catarina provavelmente está relacionada às diferenças climáticas entre ambos, e elas determinam períodos de maior atividade das aranhas, picos populacionais e períodos reprodutivos diversos. As vítimas de araneísmo, em mais de $40 \%$ dos casos, encontravam-se em atividades no interior das residências. Tais atividades, como repouso e trabalhos domésticos, são registradas como "outros" na ficha de notificação da Vigilância Epidemiológica. Esses resultados corroboram os obtidos por Málaque et al. (2002) em estudo realizado no Estado de São Paulo, onde $41 \%$ dos acidentes ocorreram em atividades domésticas, e ato de calçar os sapatos (HOSPITAL VITAL BRASIL, 2004), evidenciando novamente o hábito intradomicíliar das aranhas peçonhentas do gênero Loxosceles.
As partes do corpo mais picadas pelas aranhas foram coxas/pernas, pé/dedos e mão/dedos, com $25 \% ; 23 \%$ e $16 \%$, respectivamente, corroborando os resultados de Bucharetchi (1992). Tal acidente é ocasionado pelo fato de que aranhas do gênero Loxosceles tendem a abrigar-se da luz em sapatos, calças, roupas penduradas, roupas de cama e banho, e ao sentir-se comprimida acaba utilizando-se da picada como forma de defesa (CAMPOS; GARCIA, 1997; CUPO; AZEVEDO-MARQUES; HERING, 2003).

Quase $40 \%$ das vítimas levaram de 6 a 12 horas e mais de $20 \%$ levaram mais de 12 horas para buscar atendimento médico após a picada, diferindo das afirmações de Cardoso (1992) de que cerca de 97\% dos pacientes buscam atendimento entre 12 e 36 horas, uma vez que a dor local se agrava neste período de tempo.

As principais alterações clínicas apresentadas pelos pacientes picados pôr aranhas foram dor $(89,3 \%)$, edema $(79,3 \%)$ e eritema $(65,6 \%)$, resultados semelhantes foram obtidos por Bucharetchi (1992) e Málaque et al. (2002), no estado de São Paulo. As complicações clínicas da picada da aranha apontadas por $44 \%$ das vítimas foram mialgia, cefaléia, náuseas e vômitos , (conforme apresentado na Tabela 1), e a maioria dos casos foi classificada como leve, indo ao encontro dos resultados de Bucharetchi (1992) e Cardoso (1992). A soroterapia foi utilizada em 79,4\% dos registros, e que $93,9 \%$ dos pacientes evoluíram para cura sem sequielas. 
Tabela 1. Complicações clínicas do araneísmo no município de Chapecó, SC de 1995 a 2002, com respectivo número de pacientes e frequiência.

\begin{tabular}{lcc}
\hline \multicolumn{1}{c}{ Complicações clínicas } & Número de pacientes & Freqüência (\%) \\
\hline Hipertensão arterial sistêmica & 1 & 0,80 \\
Hipotensão & 2 & 1,5 \\
Diarréia & 5 & 3,8 \\
Náuseas e Vômitos & 14 & 10,7 \\
Choque & 1 & 0,80 \\
Dispnéia & 2 & 1,52 \\
Insuficiência Renal Aguda & 1 & 0,80 \\
Dores abdominais & 3 & 2,30 \\
Cefaléia & 14 & 10,7 \\
Mialgia & 30 & 23,0 \\
Outros (sudorese, espistaxe, oligúria e diplopia) & 19 & 14,5 \\
\hline TOTAL & $\mathbf{9 2}$ & $\mathbf{7 0 , 4 2}$ \\
\hline
\end{tabular}

\section{Conclusões}

O estudo das características clínicas e epidemiológicas do araneísmo, levado a efeito em Chapecó, SC, nas condições em que foi realizado, permite concluir que entre os acidentes com aranhas o loxoscelismo é predominante, e o araneísmo aumentou 36,3\% de 1995 a 2003, possivelmente em decorrência aumento da densidade populacional de aranhas do gênero Loxosceles por fatores antrópicos. Esses fatores podem ter favorecido o sinantropismo das mesmas, aliado ao fato da obrigatoriedade da notificação pelo Ministério da Saúde a partir de 1995, e, como se observa após o primeiro registro de Campos e Garcia (1997), a incidência tem um aumento significativo.

Verifica-se a necessidade de políticas públicas na área da saúde voltadas à orientação da população sobre os hábitos das aranhas, sinais e sintomas da picada, aparência do animal, formas de combate e prevenção dos acidentes, visto que a maioria das vítimas procuram atendimento médico entre 6 a 12 horas, o que piora o quadro clínico, e, no caso de acidentes com crianças, pode ser fatal. Assim, seriam necessárias medidas preventivas como ocorre com outros animais sinantrópicos como o Aedes aegypti, mosquito transmissor da Dengue, o que levaria à diminuição do número crescente de acidentes trazendo mais tranquiilidade à população. Sugere-se a realização de outros estudos que busquem informações sobre os acidentes sob diversos ângulos, como o conhecimento das vítimas e dos profissionais de saúde para verificar a dimensão real deste problema de saúde para este Município.

\section{Referências}

BARBARO, K. C; JARED, C; MOTA, I. Aranhas venenosas no Brasil. Revista Ciência Hoje, Rio de Janeiro, v. 19, n. 114, p. 48-52, 1995.

BARP, J. F.; GARCIA, F. R. M. Epidemiologia do araneísmo no extremo Oeste de Santa Catarina. Revista Visão Global, São Miguel do Oeste, v. 8, n. 29, p. 82-92, 2005.

BOCHNER, R.; STRUCHINER, C. J. Acidentes por animais peçonhentos e sistemas de informação. Cadernos de Saúde Pública, Rio de Janeiro, v. 18, n. 3, p. 735-746, 2002.

BRASIL. Ministério da Saúde. Manual de diagnóstico e tratamento de acidentes por animais peçonhentos. Brasília: O Ministério, 1998. 
BRASIL. Ministério da Saúde. Fundação Nacional de Saúde. Manual de diagnósticos e tratamento de acidentes por animais peçonhentos. Brasília: O Ministério, 1999.

BUCHARETCHI, F. Acidentes por Phouneutria. In: SCHVARTSMANN, S. Plantas venenosas e animais peçonhentos. 2.ed. São Paulo: Sarvier, 1992. p. 196-201.

CAMPOS, J. V.; GARCIA, F. R. M. Ocorrência da aranha marrom em áreas rurais e urbanas de Chapecó, Santa Catarina. Revista Agropecuária Catarinense, Florianópolis, v. 10, n. 3, p. 24-25, 1997.

CUPO, P.; AZEVEDO-MARQUES, M. M.; HERING, S. E. Acidentes por animais peçonhentos: escorpiões e aranhas. Medicina, Ribeirão Preto, v. 36, p. 490-497, 2003.

CARDOSO, J. L. C. Acidentes com Loxosceles. In: SCHVARTSMANN, S. Plantas venenosas e animais peçonhentos. 2.ed. São Paulo: Sarvier, 1992. p. 201-204.

HOSPITAL VITAL BRASIL. Acidentes com aranhas. Disponível em: $<$ http://www.butantan.gov.br/hvb $>$ Acesso em: 25 jul. 2004.

INSTITUTO BRASILEIRO DE GEOGRAFIA E ESTATÍSTICA - IBGE. Cidades. Disponível em: www.ibge.gov.br/cidadesat/default.php. Acesso em: 2 maio 2003.
LUCAS, S. M.; SILVA JUNIOR., P. I. Aranhas de interesse médico no Brasil. In: SCHVARTSMANN, S. Plantas venenosas e animais peçonhentos. 2.ed. São Paulo: Sarvier, 1992. p. 189-196.

MÁLAQUE, C. M. S; CASTRO-VALENCIA, J. E.; CARDOSO, J. L. C.; FRANÇA, F. O. S.; BARBARO, K. C.; FAN, H. W. Clinical and epidemilogical features of definitive and presumed loxoscelism in São Paulo, Brazil. Revista do Instituto de Medicina Tropical de São Paulo, São Paulo, v. 44, n. 3, p. 139-143, 2002.

MARQUES-DA-SILVA, E.; FISCHER, M. L. Distribuição das espécies do gênero Loxosceles Heinecken \& Lowe, 1835 (Araneae; Sicariidae) no Estado do Paraná. Revista da Sociedade Brasileira de Medicina Tropical, Uberaba, v. 38, n. 4, p.331-335, 2005.

SCHENONE, F. H. Cuadro tóxicos producidos por mordeduras de aranã en Chile: Latrodectismo y Loxocelismo. Revista Médica de Chile, Chile, v. 131, n. 4, p. 437-444, 2003. 